\title{
Mejorando los niveles de comprensión en Química a partir del enfoque de la enseñanza para la comprensión
}

\author{
Edisson Andrés Villamizar Galvis ${ }^{{ }^{*}}$ - María Tráncito Montero Ballesteros $^{2}$ \\ Miguel Antonio González Montealegre ${ }^{3}$ - María del Pilar Castillo ${ }^{4}$
}

Recepción: 14-08-2018 / Aceptación: 12-10-2018

\section{Resumen}

En la sede de secundaria de la Institución Educativa Departamental El Volcán, municipio de Ubaté, Colombia se desarrolló un proyecto de investigación acción, con enfoque cualitativo y alcance descriptivo, sobre la transformación de la práctica pedagógica para desarrollar las habilidades del pensamiento crítico como la interpretación y explicación, a partir del enfoque de la enseñanza para la comprensión.

Se analizó el proceso de transformación de la práctica pedagógica, desarrollando planteamientos curriculares que conllevaran al desarrollo de la explicación e interpretación; diseñando estrategias pedagógicas con el enfoque de la enseñanza para la comprensión, y evaluando el impacto que estas estrategias tenían sobre las habilidades del pensamiento crítico para fomentar la comprensión en los estudiantes.

Se presenta la mejora de las comprensiones de los estudiantes de grado décimo en la asignatura de Química, frente al contenido disciplinar y el desarrollo de las habilidades del pensamiento crítico mencionadas, partiendo del cambio en las prácticas pedagógicas del docente de ciencias en un ámbito rural. Por lo tanto, una organización clara y coherente en la planeación de las clases (en adelante, unidades de comprensión), las reflexiones sobre la práctica, la evaluación continua del aprendizaje y el uso de rutinas de pensamiento, conllevaron a mejorar significativamente los niveles de comprensión en los estudiantes.

Palabras clave: Práctica pedagógica, enseñanza para la comprensión, desempeños de comprensión, valoración continua, explicación e interpretación.

\section{Abstract}

In the elementary school headquarter of Institución Educativa Departamental El Volcán, city of Ubaté in Colombia, it was developed an action research project with a qualitative approach and descriptive scope about the transformation of pedagogical practice in order to create critical thinking abilities such as, interpretation and explanation based on the approach of teaching for comprehension.

It was analyzed the transformation process of the pedagogical practice that allows curricular programs which entail the development of the explanation and the interpretation. Besides, pedagogical strategies were designed to approach the teaching for comprehension and evaluate their impact on critical thinking abilities in order to promote comprehension among students.

It presents the improvement of the conditions of the tenth grade students in the subject of Chemistry, compared to the content of the discipline and the development of critical thinking skills based on the change in the pedagogical practices of Science teachers in a rural area. Therefore, a clear and coherent organization in the planning of the classes, the reflections on the practices, the continuous evaluation of the learning, and the use of routines led to improve the levels of comprehension in the students.

Keywords: Pedagogical practice, teaching for comprehension, comprehension performance, continuous assessment, explanation and interpretation.

\footnotetext{
1 Ingeniero de Producción Biotecnológica, especialista para el desarrollo del aprendizaje autónomo, Docente Ciencias naturales - Química grado décimo, Institución Educativa Departamental El Volcán, Colombia; autor para comunicación: edian162@hotmail.com

2 Docente investigadora del área de Lenguaje grado séptimo, Institución Educativa Departamental El Volcán, Colombia

3 Docente investigador del área de Matemáticas grado noveno, Institución Educativa Departamental El Volcán, Colombia

4 Magister en Pedagogía, Asesora del trabajo de investigación, Microbióloga de la Universidad de los Andes, Docente de pregrado y posgrado de la Universidad de La Sabana, Colombia
} 


\section{Introducción}

La educación es sin duda uno de los mecanismos más efectivos para brindar a los niños, jóvenes y adolescentes oportunidades para mejorar sus condiciones de vida y su actuar dentro de la sociedad; tanto así, que los contextos de desarrollo y aprendizaje de la población estudiantil y sobre todo de aquella que hace parte de un ambiente rural, influyen enormemente para otorgar estas oportunidades y mejorar su participación como miembros activos en la sociedad actual.

Por consiguiente, a lo largo de un estudio detallado de los resultados en las pruebas SA$\mathrm{BER}^{5}$ desde los años 2012 hasta el 2016, el índice sintético de calidad educativa, por sus siglas ISCE ${ }^{6}$ y los trabajos de aula, evidenciaron en la institución educativa departamental El Volcán (IDE) ${ }^{7}$, las dificultades que tenían los estudiantes en sus procesos de aprendizaje: por ejemplo, en Química, a los estudiantes se les dificulta modelar fenómenos de la naturaleza basado en el análisis de variables, la relación entre dos o más conceptos del conocimiento científico y de la evidencia derivada de investigaciones científicas ${ }^{8}$; igualmente interpretar y explicar conceptos básicos de ciencias o resolver problemas aplicando el conocimiento científico (ICFES, 2014).

Por lo tanto, haciendo un análisis documental del Proyecto Educativo Institucional (PEI) de la IED, se reconoció que se implementaba una metodología tradicional en la enseñanza de las Ciencias, porque su objetivo principal era que el estudiante memorizara los contenidos e intentara encontrar alguna conexión con el entorno o con el mundo, pero desde las planeaciones de clase no se daban pautas claras y no se tenían metas para alcanzar. Asimismo, la enseñanza no tenía presente los ritmos de aprendizaje de cada uno de los estudiantes, siendo siempre lo más importante la evaluación final del contenido y las notas que se obtuvieran al final del proceso de aprendizaje. Por consiguiente, el aprendizaje se daba de una manera superficial enfocada en la memorización y prácticas rutinarias.

Por lo tanto, era imperativo hacer cambios e implementar procesos pedagógicos diferentes dentro de la práctica, para mejorar el desempeño académico de los estudiantes y fortalecer su pensamiento crítico en las habilidades de explicación e interpretación. De igual forma, estas habilidades se relacionan

\footnotetext{
5. Pruebas SABER: son pruebas que contribuyen al mejoramiento de la calidad de la educación colombiana, mediante la realización de evaluaciones aplicadas periódicamente para monitorear el desarrollo de las competencias fundamentales en los estudiantes de educación básica y media, como seguimiento de calidad del sistema educativo. (Ministerio de Educación Nacional, 2016).

6. Es la herramienta que permite evaluar de 1 a 10 el proceso educativo de los colegios, con el fin de poder determinar los planes y acciones que se deberán llevar a cabo para lograr el mejoramiento y excelencia educativa en Colombia. (MEN, 2016).

7. Dentro del documento, los autores también se referirán a esta institución mediante su acrónimo.

8. Reporte de resultados por aplicación del examen Saber 11 para establecimientos educativos, teniendo como indicador que el 70\% de los estudiantes respondieron incorrectamente el aprendizaje evaluado en procesos químicos.
} 
directamente con las competencias en ciencias naturales como la indagación, la explicación de fenómenos y el uso comprensivo del conocimiento científico.

Con referencia a lo anterior, el desarrollo de las habilidades del pensamiento crítico como la explicación e interpretación, según Ritchhart, R. Mark, C. y Karin, M. (2014) son vistas como "movimientos del pensamiento de alto nivel que permiten desarrollar bien la comprensión" (p.26). Es así como Ron Ritchhart y sus colegas David Perkins, Shari Tishman y Patricia Palmer plantearon un mapa de pensamiento e identificaron los pensamientos esenciales para desarrollar la comprensión, los cuales son: "1. Observar de cerca y describir qué hay ahí. 2. Construir explicaciones e interpretaciones. 3. Razonar con evidencia. 4. Establecer conexiones. 5. Tener en cuenta diferentes puntos de vista y perspectivas. 6. Captar lo esencial y llegar a conclusiones" (Ritchhart, Church y Morrison, 2014, p.46).

Por ello, construir explicaciones e interpretaciones es -según los autores- un movimiento del pensamiento de alto nivel que desempeña un papel importante para generar nuevas ideas, para hacer descripciones por partes $\mathrm{y}$ completas de lo que se está aprendiendo; analizar, identificar y organizar la información recibida en el aula. En conclusión: "El proceso de comprensión está integralmente vinculado a la construcción de nuestras explicaciones e interpretaciones" (Ritchhart, et al. 2014, p.26).

Por otro lado, la mayoría de los estudiantes de la IED, después de pasar por un largo proceso de aprendizaje de muchos años en la escuela, no recuerdan ni comprenden gran parte de lo que les enseñaron; y en múltiples ocasiones el aprendizaje adquirido y la enseñanza recibida no tienen un sentido común en la vida o en el mundo real del estudiante. Con relación a lo anterior, Martha Stone (2008) indica que los estudiantes deben "aprender haciendo" (p.23); es decir, desarrollar desempeños que los encaminan hacia la comprensión y en gran medida a hacer conexiones entre lo que aprenden y lo que viven en su contexto.

Por consiguiente, en esta investigación se aplicaron estrategias pedagógicas en el aula basadas en la comprensión; pero, ¿qué es la comprensión? Uno de los principales exponentes sobre el tema es David Perkins, quien propone que es una "capacidad de desempeño flexible" o "la habilidad de pensar y actuar con flexibilidad a partir de lo que uno sabe" (citado en Stone, 2008, p. 37). Esto significa que comprender, es ir más allá del simple conocimiento de las cosas; no es solamente captar toda la información posible o responder una serie de cuestionarios de manera perfecta demostrando lo que se sabe, sino es encontrarle un verdadero sentido y significado de lo que se ha aprendido.

Por su parte, Perkins define la comprensión como "la capacidad de pensar y actuar con flexibilidad" (citado en Stone, 2008, p. 37). Él mismo indica que el contraste a esta definición es "cuando un estudiante no puede ir más allá de la memorización y el pensamiento y la acción rutinaria, esto indica falta de 
comprensión" (citado en Stone, 2008, p. 37). En este mismo sentido, parte de los desafíos actuales de la educación es propender por una pedagogía de la comprensión, que rompa las barreras que se sigue presentando en la enseñanza tradicional, que conlleve a los estudiantes al desarrollo de su pensamiento y a los docentes a plantear mallas curriculares atractivas e innovadoras, aplicando procesos de valoración acordes y con criterios claros sobre lo que se enseña.

Por otra parte, Vito Perrone asegura que "una pedagogía de la comprensión debe comprometer la gama completa de posibilidades intelectuales para que los estudiantes puedan aplicar todos sus talentos en el trabajo escolar. Más aún, debe ser adaptable a todas las materias y niveles" (citado en Stone, 2008, p. 65). Por esta razón, la investigación implementó el marco de la enseñanza para la comprensión (por su acrónimo, EpC) ${ }^{9}$ en las unidades de comprensión, con el fin de que esta "planeación”, tomara un rumbo más centrado, más claro y definido hacia lo que realmente vale la pena que los estudiantes comprendan.

De esta manera, el marco de la EpC se diseña para orientar las prácticas docentes y las estrategias pedagógicas, así como la reflexión continua del trabajo en el aula. Está planteado según Stone (2008) en cuatro partes importantes cuyos elementos son: "tópicos generativos, hilos conductores - metas de comprensión, desempeños de comprensión y evaluación diagnóstica continua” (p. 95).
Una vez delimitados los tópicos generativos, se dirige el conocimiento hacia lo que se espera que los estudiantes comprendan. Para Tina Blythe y David Outerbridge, citados por Blythe, (1999) "las metas son los conceptos, procesos y habilidades que deseamos que comprendan los estudiantes" (p. 66).deseamos que comprendan los estudiantes" (p. 66).

\section{Metodología}

Para esta investigación se plantearon tres (3) unidades de comprensión durante un año escolar, cada una con las cuatro fases del marco utilizado, las cuales son:

- Tópicos generativos: son los elementos que guían la construcción de currículos y el aprendizaje hacia el camino de la comprensión.

- Las metas: son los conceptos, procesos y habilidades que deseamos que comprendan los estudiantes; se pueden plantear en forma de pregunta o en afirmaciones dentro de una planeación curricular.

- Los desempeños de comprensión: son las actividades o tareas verdaderas que los estudiantes deben realizar para que demuestren sus habilidades y logren alcanzar las metas planteadas. Se dividen en etapa de exploración, investigación guiada y proyecto final de síntesis.

- Evaluación diagnóstica contínua: es un proceso de retroalimentación que se realiza cuando los estudiantes van desarrollando los desempeños de comprensión, de tal

\footnotetext{
9. A partir de esta acotación, los autores se referirán a este término mediante su acrónimo.
} 
forma que se brinda el apoyo para resolver dudas, inquietudes, sugerencias, correcciones y demás elementos que encaminan al estudiante a la mejora continua en su trabajo y en sus próximos desempeños.

Desde las unidades de comprensión en Química, se planearon metas explícitas para que los estudiantes alcanzaran la asimilación en contenido (propio de la disciplina), en métodos (cómo se demuestra lo que se conoce y se sabe); en propósitos (conexiones reflexivas y personales del conocimiento) y en comunicación (variedad y fluidez para comunicar el conocimiento).

De igual forma, para los desempeños de comprensión, se implementaron rutinas de pensamiento como herramientas que promueven y apoyan el desarrollo de esta en los estudiantes. Es así, que el docente investigador aplicó las siguientes rutinas de pensamiento ${ }^{10}$ :

Para presentar y explorar ideas: Ver, pensar, preguntarse; pensar, inquietar, explorar; juego de la explicación.

Para sintetizar y organizar ideas: Color, símbolo, imagen $\mathrm{CSI}^{11}$; generar, clasificar, conectar, elaborar: mapas conceptuales.

En el mismo sentido, la evaluación diagnóstica se aplicó en: los desempeños exploratorios como evaluaciones informales sobre el proceso de comprensión de los estudiantes que no requieren registrarse formalmente, en los desempeños de investigación guiada y de proyecto final de síntesis. La evaluación era más formal; se usaban criterios claros y en muchos casos los estudiantes igualmente formularon criterios y los aplicaban entre sípara evaluar su progreso en la comprensión y alcance de metas. Particularmente, para esta investigación se diseñaron rúbricas de evaluación formal con el objetivo de valorar los desempeños del proyecto final de síntesis. Particularmente, para esta investigación se diseñaron rúbricas de evaluación formal con el objetivo de valorar los desempeños del proyecto final de síntesis.

Igualmente, se planteó un ejercicio de observación, retomando las afirmaciones de Santelices (1989), en donde observar es equivalente a las acciones que realiza el hombre para desentrañar los misterios que el mundo fenomenológico encierra. En la figura 1 se observan los criterios tomados para el desarrollo de este estudio.

Una vez identificada la problemática, se determinó la siguiente pregunta de investigación: ¿cómo la transformación de la práctica pedagógica con base en el enfoque de la EpC, contribuye a fomentar el desarrollo de la interpretación y explicación en los estudiantes para que logren alcanzar mejores comprensiones?

De igual forma se realizaron reflexiones sobre el trabajo docente en el aula, basado en la comprensión y el desarrollo del pensamiento críti-

10. Según Ritchhart, et al. (2014), las rutinas son procedimientos, procesos o patrones de acción que se utilizan de manera repetitiva para manejar y facilitar el logro de metas o tareas específicas (p.54).

11. Estrategia didáctica basada en la mnemotécnica, que orienta al diagnóstico del Contexto (C), Sujeto (S) e Instrumentos (I) en la evaluación educativa. 


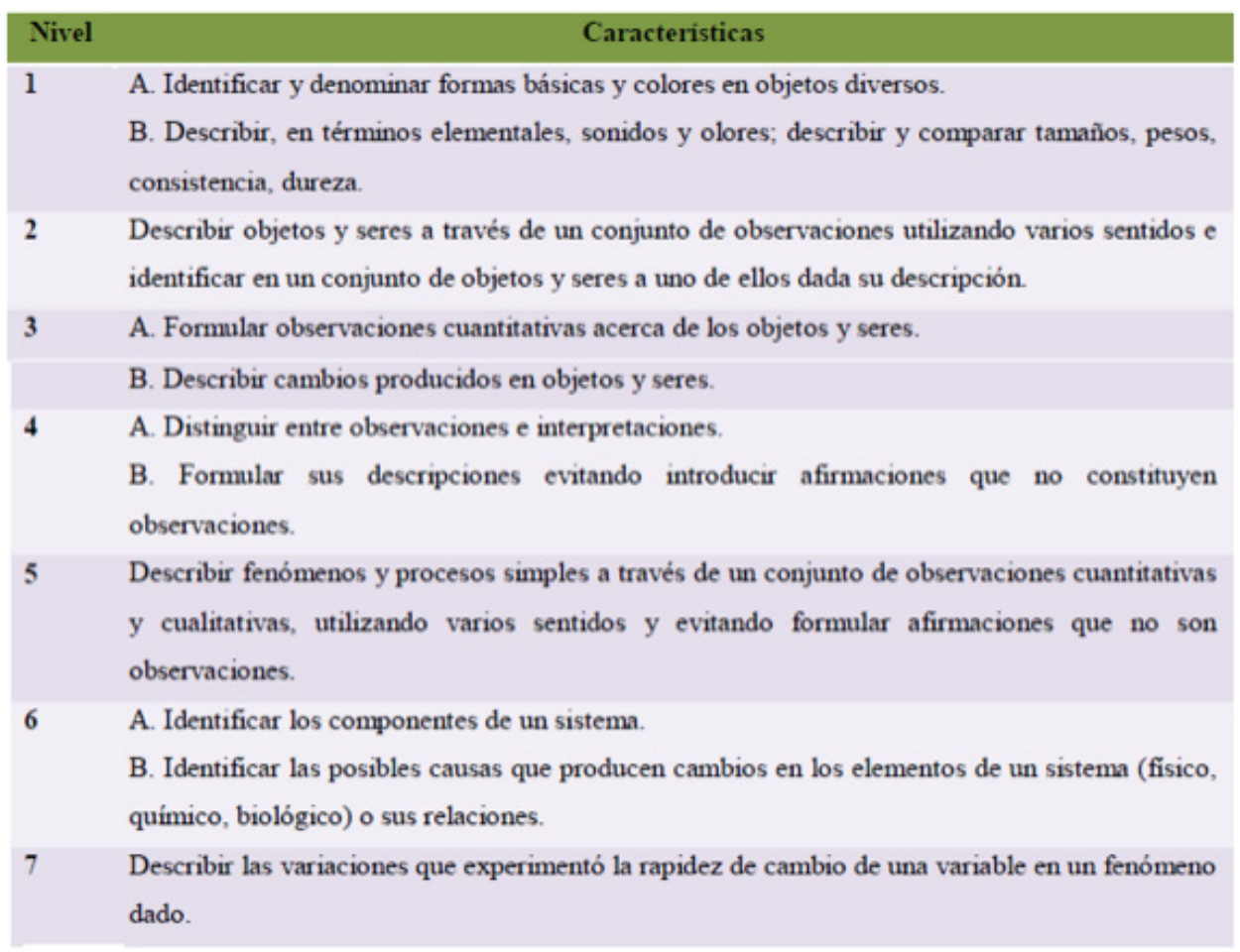

Figura 1. Niveles de observación

Fuente: Santelices, L. (1989 p. 28)

co. A partir de estos criterios se consideraron tres categorías de análisis en el transcurso de la investigación: Práctica pedagógica, comprensión y pensamiento crítico. Este artículo presenta el análisis realizado por el docente en la categoría de comprensión en Química.

La investigación desarrollada tuvo como base el enfoque cualitativo, debido a que se realizaron diversas reflexiones en cada etapa de la investigación, sobre los cambios en las prácticas pedagógicas de los docentes involucrados y de las transformaciones en los desempeños de los estudiantes. Tuvo un alcance descriptivo porque examinó qué elementos del ejercicio docente se iban modificando en la medida que se llevaba a cabo la transformación de las prácticas pedagógicas $y$, como a su vez, iban incidiendo en las habilidades del pensamiento crítico de los estudiantes para obtener mejores comprensiones.

Todo esto se realizó mediante un diseño metodológico de investigación acción pedagógica que buscó hacer una reflexión sistemática sobre la práctica docente. Asimismo, la población objeto de estudio fueron los estudiantes que se encontraban en el contexto del aula. Se recolectó información primaria a través de instrumentos como: las unidades de comprensión, las reflexiones del trabajo pedagógico, diarios de campo y las rúbricas de evaluación que ayudaron a determinar los niveles de comprensión como medio para valorar los avances en el aprendizaje de los estudiantes. 


\begin{tabular}{|c|c|c|}
\hline Categorías & Subcategorías & $\begin{array}{l}\text { Instrumentos de recolección de } \\
\text { información }\end{array}$ \\
\hline \multirow[b]{2}{*}{ Práctica pedagógica } & Planeación & $\begin{array}{l}\text { Formatos de planeación, diarios de } \\
\text { campo, unidades de comprensión. }\end{array}$ \\
\hline & Transformación curricular & $\begin{array}{l}\text { Filmaciones, observación, diario } \\
\text { de campo, reflexiones Formatos o } \\
\text { actividades en el aula. }\end{array}$ \\
\hline \multirow{3}{*}{ Comprensión } & Área de lenguaje & \multirow{3}{*}{$\begin{array}{c}\text { Rutinas de pensamiento, rúbricas } \\
\text { de valoración, fotos, observacio- } \\
\text { nes de clases }\end{array}$} \\
\hline & Área de matemáticas & \\
\hline & Área de ciencias naturales & \\
\hline \multirow[b]{2}{*}{ Pensamiento crítico } & Habilidad de interpretación & \multirow{2}{*}{$\begin{array}{l}\text { Rutinas de pensamiento, rúbricas } \\
\text { de valoración, fotos, trabajos de } \\
\text { los estudiantes }\end{array}$} \\
\hline & Habilidad de explicación & \\
\hline
\end{tabular}

Figura 2. Categorías, subcategorías e instrumentos de recolección de información.

En la figura 2, se muestra en detalle la estructura en la cual se basó el trabajo de investigación.

De acuerdo con las consideraciones anteriores y con los criterios institucionales de la IED El Volcán en la asignatura de Química, se diseñaron tres unidades de comprensión durante tres trimestres académicos. Estas unidades están basadas en los cuatro elementos de la EpC antes mencionados. De esta forma, en Química se crearon tópicos para cada unidad como: "El jabón, un producto de la genialidad humana" para el tema de estequiometria; "Los gases como medio para impulsar cohetes" para el tema de las leyes de los gases; e "Insecticida orgánico como solución contra plagas que atacan cultivos" para el tema de soluciones químicas, teniendo en cuenta que deben ser "centrales para un dominio o disciplina, interesantes para los estudiantes y el docente, rico en conexiones con otras disciplina" (Blythe, 1999, p.97).

También se planearon cuatro metas, cada una enfocada a contenidos, métodos, propósitos $\mathrm{y}$ formas de comunicar lo aprendido. Estas se establecieron en formas de preguntas como, por ejemplo: ¿Cómo interpretar las concepciones teóricas de las soluciones para crear un insecticida orgánico que contribuya a la protección de los cultivos y del medio ambiente en mi vereda? enfocada a contenidos; ¿Cómo explicar la concentración físico - química de un insecticida orgánico creado en clase como parte de una solución química? enfocada a métodos; ¿Por qué es importante usar insecticidas orgánicos en nuestra vereda? enfocada a propósitos y ¿Se puede generar una conciencia ecológica en la vereda al informar sobre el uso e implementación de productos orgáni- 
cos para el cuidado de los cultivos? enfocada a formas de comunicación.

"Las metas definen de manera más específica las ideas, procesos, relaciones o preguntas que los estudiantes comprendan por medio de su indagación" (Stone, 2008, p. 98.).

En cuanto a los desempeños de comprensión, el docente investigador propuso rutinas de pensamiento; ejercicios de observación y descripción, experimentos y proyectos finales como: la producción de jabón o la elaboración de un insecticida orgánico, relacionados a los tópicos generativos de las unidades de comprensión.

En este orden de ideas, los cuatro elementos del marco de la EpC interactúan entre sí durante todo el proceso de planificación de una unidad de comprensión; por tal motivo, la comprensión final de los estudiantes puede describirse en cuatro niveles de comprensión: ingenua, de principiante, de aprendiz y de maestría (citados en Stone, 2008).

Por consiguiente, según Verónica Mansilla y Howard Gardner, citados por Stone (2008) "los cuatro niveles de comprensión del marco de la EpC ilustran la naturaleza multidimensional de la comprensión, la capacidad de usar el conocimiento en todas las dimensiones" (p.239). (Ver figura 3).

En este mismo sentido, los niveles de comprensión se asociaron en esta investigación a los criterios institucionales de evaluación por desempeño. Es decir, el nivel ingenuo se enlaza con el desempeño bajo; el nivel principiante con el desempeño básico, el nivel aprendiz con el desempeño alto y el nivel maestría con el desempeño superior. Pero para efectos de la investigación se manejaron los niveles de comprensión según el marco de la EpC.

Por consiguiente, para el caso de la evaluación diagnóstica continua, se aplicó de manera informal en los desempeños exploratorios y de investigación guiada, de tal forma que se brindó apoyo al estudiante para resolver dudas, inquietudes, sugerencias, correcciones y demás elementos que los encaminaran a la mejora continua en su trabajo y en sus próximos desempeños.

Igualmente, se aplicó de manera formal, por medio de la retroalimentación escrita en el desempeño de proyecto final de síntesis, teniendo en cuenta que se diseñaron rúbricas de valoración que determinaban sus niveles de comprensión frente a los tópicos vistos. Estas rúbricas se hicieron públicas tanto para estudiantes como para los padres de familia, con el fin de que tuvieran en cuenta el proceso de evaluación en el aprendizaje y alcance de metas de comprensión. En la figura 4 se describen los criterios aplicados para el proyecto final de síntesis en las unidades de comprensión (ver figura 4).

El desarrollo de esta investigación se llevó a cabo directamente en el contexto de aula de clase de la asignatura de Química en el grado décimo, conformado por 25 estudiantes cuyas edades oscilan entre los 14 y 17 años de edad; pertenecientes a estratos socioeconómicos 0,1 y 2, de familias tradicionales conformada por la pareja y uno o más hijos. Es un curso que 


\begin{tabular}{|c|c|c|c|}
\hline $\begin{array}{c}\text { Comprensión } \\
\text { ingenua }\end{array}$ & $\begin{array}{l}\text { Comprensión } \\
\text { de principiante }\end{array}$ & ión & de ma \\
\hline $\begin{array}{l}\text { Los desempeños están } \\
\text { arraigados en el conoci- } \\
\text { miento intuitivo. } \\
\text { Los alumnos describen } \\
\text { la construcción de co- } \\
\text { nocimiento como un } \\
\text { proceso no problemático } \\
\text { que consiste en captar } \\
\text { información que está di- } \\
\text { rectamente disponible en } \\
\text { el mundo. } \\
\text { Los alumnos no ven la re- } \\
\text { lación entre lo que apren- } \\
\text { den en la escuela y su vida } \\
\text { cotidiana. No consideran } \\
\text { los propósitos y usos del } \\
\text { conocimiento. } \\
\text { Los desempeños no } \\
\text { muestran signos de do- } \\
\text { minio, por parte de los } \\
\text { alumnos, de lo que saben. } \\
\text { Los desempeños son no } \\
\text { reflexivos respecto de las } \\
\text { formas en las que el co- } \\
\text { nocimiento se expresa o } \\
\text { comunica a los demás. }\end{array}$ & $\begin{array}{l}\text { Los desempeños se arrai- } \\
\text { gan en los rituales de prue- } \\
\text { bas y de la escolarización. } \\
\text { Los alumnos empiezan a } \\
\text { interpolar algunos con- } \\
\text { ceptos o ideas disciplina- } \\
\text { rias y a establecer cone- } \\
\text { xiones simples, a menudo } \\
\text { ensayadas entre ellos. } \\
\text { Los alumnos descri- } \\
\text { ben la naturaleza y los } \\
\text { propósitos de la cons- } \\
\text { trucción del conoci- } \\
\text { miento, así como su } \\
\text { comunicación como } \\
\text { procedimientos mecá- } \\
\text { nicos paso por paso. } \\
\text { La validación de los pro- } \\
\text { cedimientos de construc- } \\
\text { ción de conocimiento } \\
\text { depende de la autoridad } \\
\text { externa más que de crite- } \\
\text { rios racionalmente con- } \\
\text { sensuados desarrollados } \\
\text { dentro de las disciplinas } \\
\text { o dominios. }\end{array}$ & $\begin{array}{l}\text { Los desempeños están } \\
\text { arraigados en el conoci- } \\
\text { miento disciplinario y mo- } \\
\text { dalidades de pensamiento. } \\
\text { Los alumnos demuestran un } \\
\text { uso flexible de los conceptos } \\
\text { o ideas disciplinarios. } \\
\text { Los alumnos ven la construc- } \\
\text { ción del conocimiento como } \\
\text { algo complejo, que sigue } \\
\text { procedimientos y criterios } \\
\text { prototípicamente usados por } \\
\text { expertos del dominio. } \\
\text { Con apoyo, los desempe- } \\
\text { ños iluminan la relación } \\
\text { entre conocimiento disci- } \\
\text { plinario y vida cotidiana, } \\
\text { examinando las oportuni- } \\
\text { dades y las consecuencias } \\
\text { de usar este conocimiento. } \\
\text { Los desempeños demues- } \\
\text { tran una expresión y co- } \\
\text { municación flexible y ade- } \\
\text { cuada del conocimiento. }\end{array}$ & $\begin{array}{l}\text { te integradores, creativos y críticos. } \\
\text { bilidad a través de dimensiones, vincu- } \\
\text { lando los criterios por los cuales se cons- } \\
\text { truye y convalida el conocimiento en una } \\
\text { disciplina, con su objeto de estudio o los } \\
\text { propósitos de la investigación. } \\
\text { Los alumnos ven la construcción de cono- } \\
\text { cimiento como algo complejo, impulsado } \\
\text { a menudo por marcos y visiones del mun- } \\
\text { do encontrados y que surge como resulta- } \\
\text { do de una argumentación pública dentro } \\
\text { de las comunidades de profesionales en } \\
\text { diversos dominios. } \\
\text { Los alumnos pueden usar el conocimien- } \\
\text { to para reinterpretar y actuar en el mun- } \\
\text { do que los rodea. } \\
\text { El conocimiento es expresado y comuni- } \\
\text { cado a otros de manera creativa. } \\
\text { Los desempeños a menudo van más allá de } \\
\text { demostrar comprensión disciplinaria para } \\
\text { reflejar la conciencia crítica de los alumnos } \\
\text { acerca de la construcción del conocimiento } \\
\text { en los dominios (por ejemplo, la compren- } \\
\text { sión metadisciplinaria) o la capacidad de } \\
\text { los alumnos para combinar disciplinas en } \\
\text { sus tareas (por ejemplo, comprensión inter- } \\
\text { disciplinaria). }\end{array}$ \\
\hline
\end{tabular}

Figura 3. Niveles de comprensión

Fuente: Hetland, L.; Hammerness, K.; Unger, C. y Gray W., D. (citados en Stone, 2008. p. 262

no presenta mayores dificultades frente a la disciplina; sin embargo, existen problemáticas sociales marcadas en la vida de ellos como la pobreza, embarazos no planificados y demás conflictos familiares que afectan en ocasiones su comportamiento y situaciones académicas.

\section{Resultados}

Los resultados alcanzados en esta investigación se obtuvieron de acuerdo al trabajo docente realizado a partir de las tres categorías de análisis desarrolladas: práctica pedagógica, comprensión y pensamiento crítico, y las subcategorías en cada una: planeación, transformación curricular; comprensiones desde cada asignatura y habilidades del pensamiento crítico como la explicación e interpretación. Respectivamente a partir de tres unidades de comprensión, aplicadas en el aula durante tres trimestres académicos, teniendo en cuenta los criterios institucionales de la IED El Volcán. 


\begin{tabular}{|c|c|c|c|c|}
\hline $\begin{array}{l}\text { CRITERIOS / NIVE- } \\
\text { LES DE COMPREN- } \\
\text { SIÓN - APRENDIZAJE }\end{array}$ & INGENUO (BAJO) & $\begin{array}{c}\text { PRINCIPIANTE } \\
\text { O NOVATO } \\
\text { (BÁSICO) }\end{array}$ & $\begin{array}{l}\text { APRENDÍZ } \\
\text { (ALTO) }\end{array}$ & $\begin{array}{l}\text { MAESTRÍA } \\
\text { (SUPERIOR) }\end{array}$ \\
\hline $\begin{array}{l}\text { Interpreta las concep- } \\
\text { ciones teóricas de las } \\
\text { soluciones en la prepa- } \\
\text { ración del insecticida } \\
\text { orgánico preparado. }\end{array}$ & $\begin{array}{l}\text { Tiene nociones sobre } \\
\text { los conceptos y apro- } \\
\text { ximaciones teóricas de } \\
\text { las soluciones dificul- } \\
\text { tándose la preparación } \\
\text { correcta del insecticida. }\end{array}$ & $\begin{array}{l}\text { Hace deducciones a } \\
\text { partir de la información } \\
\text { que describe a las solu- } \\
\text { ciones dificultándose } \\
\text { la preparación correcta } \\
\text { del insecticida. }\end{array}$ & $\begin{array}{l}\text { Interpreta la informa- } \\
\text { ción que describe las } \\
\text { soluciones logrando } \\
\text { preparar el insectici- } \\
\text { da orgánico. }\end{array}$ & $\begin{array}{l}\text { Interpreta y relaciona } \\
\text { la información que } \\
\text { describe las solucio- } \\
\text { nes para preparar co- } \\
\text { rrectamente el insec- } \\
\text { ticida orgánico. }\end{array}$ \\
\hline $\begin{array}{l}\text { Explica la concentración } \\
\text { físico - química del in- } \\
\text { secticida orgánico prepa- } \\
\text { rado, como parte de una } \\
\text { solución química. }\end{array}$ & $\begin{array}{l}\text { No da razones de la } \\
\text { concentración físico } \\
\text { - química de las so- } \\
\text { luciones para la pre- } \\
\text { paración del insecti- } \\
\text { cida orgánico. }\end{array}$ & $\begin{array}{l}\text { No logra dar explicacio- } \\
\text { nes precisas de la concen- } \\
\text { tración físico - química } \\
\text { de las soluciones para la } \\
\text { preparación del insectici- } \\
\text { da orgánico. }\end{array}$ & $\begin{array}{l}\text { Da explicaciones bási- } \\
\text { cas sobre la concentra- } \\
\text { ción físico - química } \\
\text { de las soluciones para } \\
\text { la preparación del in- } \\
\text { secticida orgánico. }\end{array}$ & $\begin{array}{l}\text { Da explicaciones pre- } \\
\text { cisas y analíticas so- } \\
\text { bre la concentración } \\
\text { físico - química de } \\
\text { las soluciones para la } \\
\text { preparación del in- } \\
\text { secticida orgánico. }\end{array}$ \\
\hline $\begin{array}{l}\text { Cumple funciones cuan- } \\
\text { do se trabaja en equipo } \\
\text { para la elaboración del } \\
\text { insecticida orgánico y su } \\
\text { aplicación en los cultivos } \\
\text { de la vereda. E identifica } \\
\text { la importancia de usar } \\
\text { un insecticida orgánico } \\
\text { en sus cultivos }\end{array}$ & $\begin{array}{l}\text { No establece ni cum- } \\
\text { ple funciones dentro } \\
\text { del trabajo en equipo } \\
\text { tanto en la elabora- } \\
\text { ción como en la apli- } \\
\text { cación del insecticida } \\
\text { orgánico. No le ve la } \\
\text { aplicación de usar in- } \\
\text { secticidas orgánicos. }\end{array}$ & $\begin{array}{l}\text { Cumple algunas funcio- } \\
\text { nes dentro del trabajo } \\
\text { en equipo tanto en la } \\
\text { elaboración como en la } \\
\text { aplicación del insecticida } \\
\text { orgánico. Identifica algu- } \\
\text { na importancia del uso de } \\
\text { insecticidas orgánicos. }\end{array}$ & $\begin{array}{l}\text { Establece y cumple } \\
\text { funciones sencillas } \\
\text { dentro del trabajo en } \\
\text { equipo tanto en la ela- } \\
\text { boración como en la } \\
\text { aplicación del insecti- } \\
\text { cida orgánico. Identi- } \\
\text { fica la importancia de } \\
\text { usar la mayor parte } \\
\text { insecticidas orgánicos } \\
\text { en sus cultivos. }\end{array}$ & $\begin{array}{l}\text { Establece y cumple } \\
\text { claramente funciones } \\
\text { dentro del trabajo en } \\
\text { equipo tanto en la ela- } \\
\text { boración como en la } \\
\text { aplicación del insectici- } \\
\text { da orgánico. Identifica } \\
\text { la importancia de usar } \\
\text { siempre insecticidas or- } \\
\text { gánicos en sus cultivos. }\end{array}$ \\
\hline $\begin{array}{l}\text { Escribe adecuadamen- } \\
\text { te en la etiqueta del } \\
\text { insecticida orgánico, la } \\
\text { información química } \\
\text { que lo describe. }\end{array}$ & $\begin{array}{l}\text { No expresa de forma } \\
\text { adecuada la infor- } \\
\text { mación química que } \\
\text { describe el contenido } \\
\text { del insecticida orgá- } \\
\text { nico preparado. }\end{array}$ & $\begin{array}{l}\text { Expresa con dificultad la in- } \\
\text { formación química que des- } \\
\text { cribe el contenido del insec- } \\
\text { ticida orgánico preparado. }\end{array}$ & $\begin{array}{l}\text { Expresa la informa- } \\
\text { ción química que des- } \\
\text { cribe el contenido del } \\
\text { insecticida orgánico } \\
\text { preparado con algunos } \\
\text { errores por corregir. }\end{array}$ & $\begin{array}{l}\text { Expresa correctamente } \\
\text { la información quími- } \\
\text { ca que describe el con- } \\
\text { tenido del insecticida } \\
\text { orgánico preparado. }\end{array}$ \\
\hline
\end{tabular}

Figura 4. Rúbrica de valoración.

No obstante, para efectos de este artículo se presentan únicamente los resultados obtenidos en la categoría de comprensión, a partir del análisis y la reflexión docente de la planeación establecida durante el año escolar (ver figura 5).

Teniendo en cuenta los tres desempeños valorados: exploratorio $(\mathrm{DE})$, investigación guiada (DIG) y proyecto final de síntesis (DPF), se muestran los resultados en la figura 6 .
De acuerdo a la figura 6, en el exploratorio se realizaron ejercicios de observación ya que es una habilidad propia del pensamiento científico, y se evaluó teniendo en cuenta los niveles de observación de Santelices (1989) ${ }^{12}$. Para la investigación guiada se trabajaron diversas rutinas de pensamiento y en los proyectos finales de tesis se desarrollaron actividades de aula relacionadas a los tópicos generativos.

12. Santelices (1989) afirma que el observar para el niño, como proceso científico, es equivalente a las acciones que realiza el hombre de ciencias. Por lo tanto, reconoció siete niveles de observación. 


\begin{tabular}{|c|c|c|c|c|c|c|}
\hline 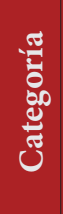 & 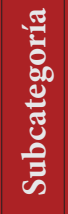 & $\begin{array}{c}\text { Estrategias ini- } \\
\text { ciales }\end{array}$ & 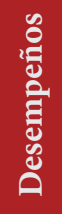 & $\begin{array}{c}\text { Unidad de } \\
\text { Comprensión } 1\end{array}$ & $\begin{array}{c}\text { Unidad de } \\
\text { Comprensión } 2\end{array}$ & $\begin{array}{c}\text { Unidad de } \\
\text { Comprensión } 3\end{array}$ \\
\hline 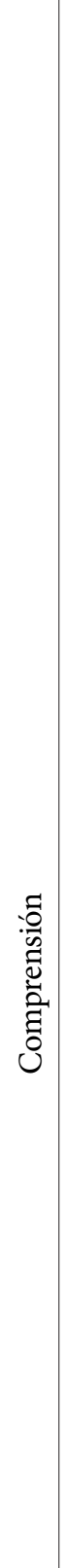 & 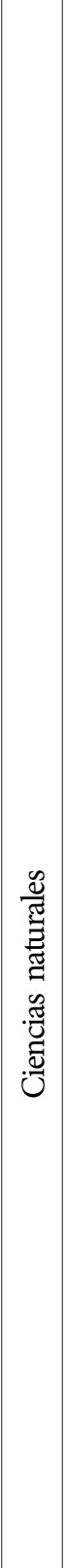 & $\begin{array}{l}\text { Anteriormente, } \\
\text { esta asignatura se } \\
\text { enseñaba ciencias } \\
\text { desde el punto de } \\
\text { vista de contenidos } \\
\text { sin tener en cuenta } \\
\text { las habilidades y } \\
\text { competencias es- } \\
\text { pecíficas de la asig- } \\
\text { natura, no existía } \\
\text { una conexión en- } \\
\text { tre la planeación, } \\
\text { las competencias, } \\
\text { las habilidades y } \\
\text { lo que se enseña- } \\
\text { ba directamente } \\
\text { en el aula. Igual- } \\
\text { mente, realizaba } \\
\text { estrategias peda- } \\
\text { gógicas enfocadas } \\
\text { a la transmisión de } \\
\text { conocimiento, cla- } \\
\text { ses magistrales o } \\
\text { expositivas, orga- } \\
\text { nización rígida en } \\
\text { filas, los estudian- } \\
\text { tes copiaban al pie } \\
\text { de la letra lo que } \\
\text { el docente escribía } \\
\text { en el tablero, falta } \\
\text { de innovación en } \\
\text { la formulación de } \\
\text { prácticas pedagó- } \\
\text { gicas para hacer vi- } \\
\text { sible el pensamien- } \\
\text { to en cada uno de } \\
\text { los estudiantes. }\end{array}$ & 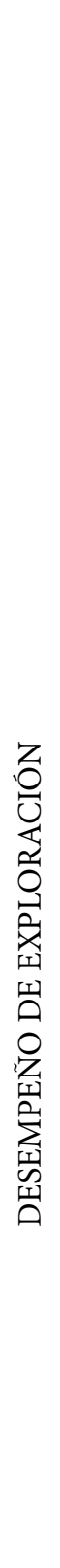 & $\begin{array}{l}\text { Se plantea un ejercicio de ob- } \\
\text { servación con los estudiantes } \\
\text { de la siguiente forma: se entrega } \\
\text { a cada estudiante un elemento } \\
\text { químico del laboratorio, estos } \\
\text { elementos están contenidos en } \\
\text { frascos rotulados y etiqueta- } \\
\text { dos; se dan instrucciones a los } \\
\text { estudiantes sobre la manipula- } \\
\text { ción correcta de estos reactivos. } \\
\text { Cada estudiante destapa el fras- } \\
\text { co, observa detenidamente su } \\
\text { contenido y hace sus descrip- } \\
\text { ciones. Los resultados de este } \\
\text { ejercicio se clasifican según los } \\
\text { niveles de observación de San- } \\
\text { telices (1989), por consiguiente, } \\
\text { se evidenció que: el 5,3\% está } \\
\text { en nivel SC (sin categoría), el } \\
\text { 47,4\% está en nivel 1A, el 10,5\% } \\
\text { en nivel 1B, el 31,6\% en nivel } \\
4 \mathrm{~B} \text { y un 5,3\% en nivel 5. Según } \\
\text { estos resultados la tendencia del } \\
\text { grupo es de nivel 1A: Identificar } \\
\text { y denominar formas básicas } \\
\text { y colores en objetos diversos. } \\
\text { Por consiguiente un gran por- } \\
\text { centaje de estudiantes } 47,4 \% \\
\text { se encuentran en un nivel de } \\
\text { comprensión ingenuo, solo } \\
\text { captan la información que al- } \\
\text { canzan a observar directamen- } \\
\text { te sin hacer ningún proceso de } \\
\text { pensamiento que les permita } \\
\text { considerar el propósito de la ac- } \\
\text { tividad. Ver anexo } 8 \text { desarrollos } \\
\text { de unidades de comprensión } \\
\text { ciencias naturales - química. }\end{array}$ & $\begin{array}{l}\text { Por medio de un ejerci- } \\
\text { cio de observación so- } \\
\text { bre los elementos de la } \\
\text { tabla periódica que cada } \\
\text { uno tenía en el libro de } \\
\text { química, debían descri- } \\
\text { bir lo que observaran } \\
\text { teniendo en cuenta sus } \\
\text { conocimientos previos } \\
\text { sobre el tema. La cla- } \\
\text { sificando este ejercicio } \\
\text { según Niveles de ob- } \\
\text { servación de Santelices } \\
\text { (1989), se evidenció } \\
\text { que: } 8,7 \% \text { tienen un ni- } \\
\text { vel Sin categoría; } 21,7 \% \\
\text { nivel 1A; } 34,8 \% \text { nivel } \\
\text { 1B; } 4,3 \% \text { nivel } 2 ; 21,7 \% \\
\text { nivel } 3 B \text { y } 8,8 \% \text { nivel } 4 \mathrm{~B} . \\
\text { Por consiguiente el gru- } \\
\text { po tiene una tendencia } \\
\text { de nivel 1B (Santelices } \\
\text { 1989) (Uno de los más } \\
\text { bajos, el máximo nivel } \\
\text { es 7) es decir describen } \\
\text { sus observaciones en } \\
\text { términos elementales } \\
\text { como colores, formas, } \\
\text { pesos y comparaciones; } \\
\text { lo que sugiere que un } \\
\text { gran porcentaje de es- } \\
\text { tudiantes } 34,8 \% \text { se en- } \\
\text { cuentran en un nivel in- } \\
\text { genuo de comprensión, } \\
\text { porque usan el conoci- } \\
\text { miento intuitivo y solo } \\
\text { captan información que } \\
\text { está directamente dis- } \\
\text { ponible a la mano. Con- } \\
\text { cluyendo que aún falta } \\
\text { desarrollar la habilidad } \\
\text { de observación en cien- } \\
\text { cias. Ver anexo } 8 \text { desa- } \\
\text { rrollos de unidades de } \\
\text { comprensión ciencias } \\
\text { naturales - química. }\end{array}$ & $\begin{array}{l}\text { El ejercicio consiste en } \\
\text { Ubicar dentro del salón } \\
\text { de clase y a la vista de } \\
\text { todos un afiche de la } \\
\text { tabla periódica de los } \\
\text { elementos químicos, } \\
\text { los estudiantes debían } \\
\text { observar y hacer des- } \\
\text { cripciones detalladas } \\
\text { de los elementos } \\
\text { Los resultados obtenidos } \\
\text { son los siguientes, según } \\
\text { Niveles de observación } \\
\text { de Santelices (1989): El } \\
20 \% \text { de los estudiantes } \\
\text { se encuentra en un nivel } \\
\text { 1A, el 75\% en nivel 3A y } \\
\text { el } 5 \% \text { en nivel } 4 \text { B. Para } \\
\text { este segundo ejercicio } \\
\text { el grupo tiene una ten- } \\
\text { dencia de observación } \\
\text { de nivel 3A: Formular } \\
\text { observaciones cuanti- } \\
\text { tativas acerca de los ob- } \\
\text { jetos y seres (Santelices } \\
1989 \text {. Por tal motivo } \\
\text { un gran porcentaje de } \\
\text { estudiantes } 75 \% \text { se en- } \\
\text { cuentran en un nivel } \\
\text { de Principiante, porque } \\
\text { comienzan a estable- } \\
\text { cer conexiones simples } \\
\text { entre el mundo que los } \\
\text { rodea y el conocimiento } \\
\text { que han adquirido hasta } \\
\text { el momento. Ver anexo } 8 \\
\text { desarrollos de unidades } \\
\text { de comprensión ciencias } \\
\text { naturales - química. }\end{array}$ \\
\hline
\end{tabular}




\begin{tabular}{|c|c|c|c|c|c|c|}
\hline 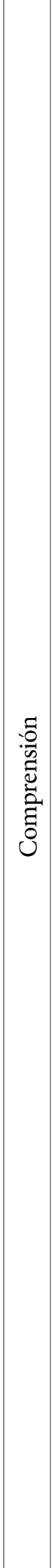 & 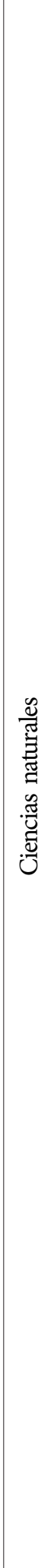 & $\begin{array}{l}\text { Los estudiantes no } \\
\text { aprendían a desa- } \\
\text { rrollar las habi- } \\
\text { lidades del pen- } \\
\text { samiento porque } \\
\text { desde la misma } \\
\text { planeación no se } \\
\text { tenía claridad del } \\
\text { objetivo de la cla- } \\
\text { se. El aprendiza- } \\
\text { je se realizaba a } \\
\text { un mismo ritmo } \\
\text { para todos, así } \\
\text { como prevalecía } \\
\text { el aprendizaje me- } \\
\text { morístico e ins- } \\
\text { truccional den- } \\
\text { tro del aula. En } \\
\text { muchos casos los } \\
\text { estudiantes no ex- } \\
\text { ponían sus ideas y } \\
\text { por ende las con- } \\
\text { cepciones erróneas } \\
\text { que adquirían so- } \\
\text { bre los contenidos } \\
\text { conllevaban a un } \\
\text { bajo nivel de su } \\
\text { aprendizaje y de su } \\
\text { comprensión. }\end{array}$ & 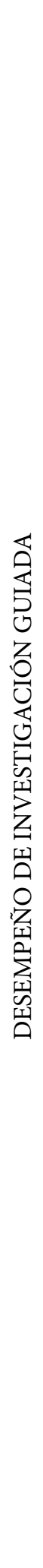 & $\begin{array}{l}\text { Se propone a los estudiantes } \\
\text { hacer un proceso de com- } \\
\text { prensión lectora a partir de } \\
\text { una lectura "las reacciones } \\
\text { químicas en la vida cotidiana", } \\
\text { con el apoyo de la rutina de } \\
\text { pensamiento "color símbolo } \\
\text { imagen CSI". Porque es una } \\
\text { rutina para sintetizar y orga- } \\
\text { nizar ideas Los resultados ob- } \\
\text { tenidos fueron los siguientes: } \\
\text { el } 20 \% \text { de los estudiantes se } \\
\text { encuentra en un nivel Prin- } \\
\text { cipiante porque solamente } \\
\text { se concentraron a seguir la } \\
\text { instrucciones pero la calidad } \\
\text { de sus metáforas no presentó } \\
\text { un nivel profundo del pensa- } \\
\text { miento; el } 20 \% \text { se encuentra } \\
\text { en un nivel Aprendiz, porque } \\
\text { hicieron procesos de pensa- } \\
\text { miento más profundos así } \\
\text { como las metáforas plantea- } \\
\text { das pero los estudiantes, re- } \\
\text { quieren de acompañamiento } \\
\text { y apoyo. El } 60 \% \text { logró hacer } \\
\text { conexiones relacionadas con } \\
\text { ideas nuevas y las que ya co- } \\
\text { nocían sin embargo hay mu- } \\
\text { chos vacíos aún con la idea } \\
\text { central de la lectura y la cons- } \\
\text { trucción profunda de metáfo- } \\
\text { ras, demostraron poco interés } \\
\text { y compromiso frente a la ru- } \\
\text { tina; por consiguiente según } \\
\text { el análisis realizado el nivel } \\
\text { de comprensión es ingenuo, } \\
\text { porque solamente captaron } \\
\text { iencias naturales - química. } \\
\text { información disponible en la } \\
\text { lectura y no profundizaron en } \\
\text { sus ideas y las elecciones son } \\
\text { muy obvias al escoger las dife- } \\
\text { rentes categorías de la rutina, } \\
\text { la calidad de las metáforas no } \\
\text { tiene un nivel más profun- }\end{array}$ & $\begin{array}{l}\text { Los estudiantes realizarán } \\
\text { una rutina de pensamien- } \\
\text { to para explorar las ideas } \\
\text { más profundamente como } \\
\text { la: AAC (Afirmar, Apoyar, } \\
\text { Cuestionar), con el fin de } \\
\text { ser críticos al detectar y } \\
\text { analizar información sobre } \\
\text { la verdad de las cosas en } \\
\text { este caso sobre la compro- } \\
\text { bación de la ley de Charles } \\
\text { en los Gases, para esto ten- } \\
\text { drán en cuenta un video y } \\
\text { una lectura sobre el tema. } \\
\text { Los resultados obtenidos } \\
\text { dan cuenta que el } 73 \% \text { de } \\
\text { los estudiantes comproba- } \\
\text { ron que la ley de Charles } \\
\text { tiene validez si se cumplen } \\
\text { con algunos criterios espe- } \\
\text { cíficos para los gases ya que } \\
\text { lograron hacer conexiones } \\
\text { entre el video (un experi- } \\
\text { mento real) y la lectura que } \\
\text { hicieron del libro. Su nivel } \\
\text { de comprensión según el } \\
\text { análisis realizado es Prin- } \\
\text { cipiante, porque los estu- } \\
\text { diantes lograron interponer } \\
\text { conceptos ya vistos frente } \\
\text { al experimento presentado } \\
\text { en el video es decir crearon } \\
\text { afirmaciones que explica- } \\
\text { ban e interpretaban ese ex- } \\
\text { perimento y establecieron } \\
\text { conexiones simples entre el } \\
\text { video y la lectura realizada. } \\
\text { Un } 4 \% \text { de los estudiantes se } \\
\text { encuentra en nivel Apren- } \\
\text { diz, porque lograron de- } \\
\text { tectar ideas que les permi- } \\
\text { tieron hacer afirmaciones } \\
\text { coherentes y explicaciones } \\
\text { acertadas y comprobar la } \\
\text { verdad del evento presen- } \\
\text { tado } \\
\text { Por otro lado el } 23 \% \text { de los } \\
\text { estudiantes presenta difi- } \\
\text { cultades para hacer visible } \\
\text { su pensamiento ya que solo } \\
\text { describen opiniones o se } \\
\text { copian de los demás com- } \\
\text { pañeros, demostraron poco } \\
\text { interés y compromiso. Por } \\
\text { lo tanto su nivel de com- } \\
\text { prensión es Ingenuo. Ver } \\
\text { anexo } 8 \text { desarollos de uni- } \\
\text { cien- } \\
\text { adea. }\end{array}$ & $\begin{array}{l}\text { Por medio de una práctica } \\
\text { de laboratorio y en grupos } \\
\text { de trabajo, los estudiantes } \\
\text { debían preparar diferentes } \\
\text { soluciones con distintos } \\
\text { tipos de concentraciones } \\
\text { tanto físicas (\%p/p, p/v) } \\
\text { como químicas (molari- } \\
\text { dad, molalidad y norma- } \\
\text { lidad) usando como mate- } \\
\text { riales básicos: agua, sal y } \\
\text { azúcar. Los resultados es- } \\
\text { perados permitieron perci- } \\
\text { bir que el } 80 \% \text { de los estu- } \\
\text { diantes lograron preparar y } \\
\text { caracterizar las soluciones } \\
\text { químicas, siguiendo al pie } \\
\text { de la letra las instruccio- } \\
\text { nes dadas; en este sentido, } \\
\text { según el análisis realizado } \\
\text { el nivel de comprensión } \\
\text { es de principiante, porque } \\
\text { los estudiantes usaron las } \\
\text { ideas y conceptos propios } \\
\text { de la disciplina para reali- } \\
\text { zar las soluciones y carac- } \\
\text { terizarlas y comunicar a } \\
\text { los demás companeros los } \\
\text { procedimientos realizados } \\
\text { paso a paso. Un } 10 \% \text { en } \\
\text { nivel Aprendiz, porque los } \\
\text { procesos de pensamiento } \\
\text { demostraron compren- } \\
\text { siones más profundas y } \\
\text { apropiación de lo aprendi- } \\
\text { do en la caracterización de } \\
\text { las propiedades químicas y } \\
\text { en la socialización de di- } \\
\text { chas soluciones y un } 10 \% \\
\text { presentó deficiencias en la } \\
\text { preparación de las caracte- } \\
\text { rísticas físicas de las solu- } \\
\text { ciones porque las prepara- } \\
\text { ron mal o simplemente no } \\
\text { las hicieron por tal motivo } \\
\text { su nivel de comprensión } \\
\text { es Ingenuo. Ver anexo } 8 \\
\text { desarrollos de unidades de } \\
\text { comprensión ciencias na- } \\
\text { turales - química. }\end{array}$ \\
\hline
\end{tabular}

Figura 5. Matriz de Química para la categoría de comprensión. 


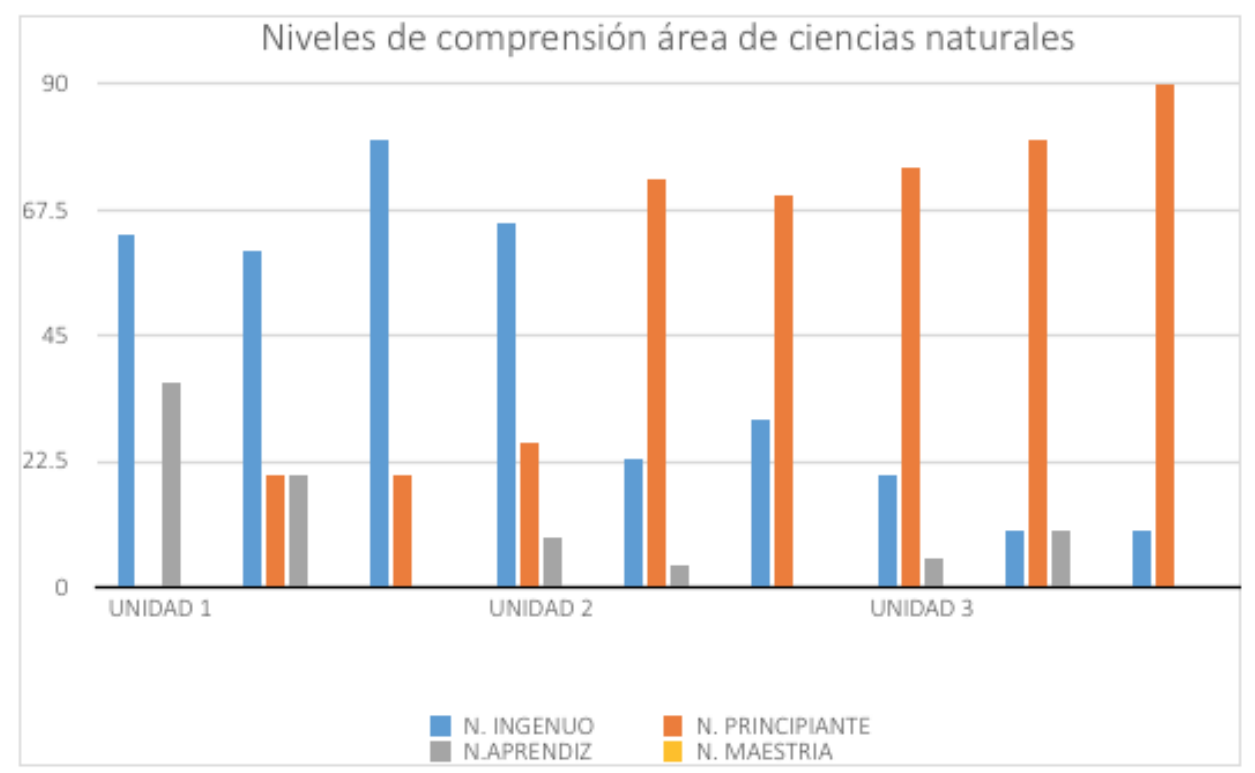

Figura 6. Niveles de comprensión en Química

El consolidado de los resultados permitió evidenciar que en la primera unidad (figura 5), más de la mitad del grupo se encontraba en el nivel de comprensión ingenuo. Para el desempeño de exploración en el ejercicio de observación, los estudiantes resolvieron esta actividad con base en conocimientos intuitivos sin acudir al razonamiento. No obstante, un porcentaje representativo del grupo alcanzó el nivel aprendiz, debido a que siguieron adecuadamente la instrucción haciendo descripciones profundas y claras de lo observado. En el desempeño de investigación guiada de la misma unidad, también hay un grupo minoritario que alcanza el nivel aprendiz, pero en el desempeño de proyecto final de síntesis ya no hay ningún estudiante, porque se pretendía que los estudiantes establecieran la relación entre el tópico y su vida cotidiana, por medio de la elaboración de jabón artesanal. Sin embargo, las unidades iniciales no eran tan claras porque presentaban algunas defi- ciencias en la conexión entre tópicos generativos, metas y desempeños de comprensión.

En la segunda unidad (figura 5), el nivel ingenuo fue disminuyendo y comenzó un aumento en el nivel de principiante a medida que se desarrollaban los desempeños de comprensión. En el desempeño de exploración, el porcentaje de estudiantes en nivel ingenuo se mantuvo prácticamente igual; y el porcentaje en nivel aprendiz se redujo casi en la mitad con respecto a la primera unidad, debido a que el ejercicio de observación propuesto en este caso estaba fundamentado en la teoría de la tabla periódica y los estudiantes no mostraban un uso flexible del conocimiento disciplinar.

En los desempeños de comprensión de investigación guiada y de proyecto final de síntesis, los estudiantes mostraron una disminución significativa en el nivel ingenuo, alcanzando el nivel de aprendiz; esto se logró gracias 
a que el docente hizo una gestión adecuada del manejo de información a través del uso de rutinas de pensamiento como: "afirmar, apoyar, cuestionar", diseñada para identificar y probar la verdad sobre afirmaciones, ideas u opiniones frente a un tema o evento adecuado para promover el pensamiento científico.

En la tercera unidad (figura 5) se logró que los niveles de comprensión ingenua disminuyeran y siguiera en aumento el nivel de principiante, ya que la adecuada gestión de los desempeños dentro de la unidad de comprensión y un ejercicio pedagógico reflexivo por parte del docente, permitieron que los estudiantes avanzaran en sus comprensiones. De igual manera, sus explicaciones lograban hacer conexiones simples entre lo que sabían y el mundo que los rodea; y las interpretaciones que realizaban daban cuenta del propósito de su aprendizaje, pero aún faltó desarrollar en ellos comprensiones más profundas para alcanzar niveles de maestría en las habilidades del pensamiento.

\section{Discusión}

La práctica pedagógica pasa de ser un ejercicio meramente rutinario a un ejercicio profesional, analítico y reflexivo, por medio de una organización clara y coherente en el diseño de unidades de comprensión estructuradas bajo los parámetros de la EpC. Durante este estudio se logró determinar que los desempeños aplicados son propicios para que los estudiantes hagan conexiones entre lo que aprenden y lo que viven en su contexto., tal como lo menciona Martha Stone (2008) cuando indica que los estudiantes deben "aprender haciendo". Por otro lado, David Perkins, propone que la comprensión es una "capacidad de desempeño flexible" o "es la habilidad de pensar y actuar con flexibilidad a partir de lo que uno sabe" (citado en Stone, 2008, p. 37). Por consiguiente, los estudiantes mejoraron sus niveles de comprensión cuando lograron pensar y actuar flexiblemente en el desarrollo de los proyectos finales de síntesis como en la elaboración del jabón, la preparación de un insecticida orgánico y la participación en la feria de la ciencia con los cohetes impulsados por gases. De esta forma pueden encontrarle un verdadero sentido a todo lo que aprenden en la escuela.

En consecuencia, el diseño de planeaciones docentes usando las cuatro fases de la EpC, promueven el desarrollo de la comprensión en el aprendizaje de los estudiantes. De tal forma, Tina Blythe y David Perkins indican que los tópicos generativos tienen características claves: "son centrales para una o más disciplinas y resultan atractivos para los estudiantes" (citados en Blythe, 1999 p. 44). En esta investigación los tópicos utilizados en las unidades de comprensión llamaron la atención, promovieron el interés, la curiosidad y la motivación en los estudiantes por alcanzar las metas propuestas.

En este mismo sentido, Tina Blythe y David Outerbridge proponen que: las metas de comprensión, conocidas como metas de comprensión abarcadoras o hilos conductores, especifican cuánto deseamos que los estudiantes obtengan de su trabajo con nosotros a lo largo de un semestre o de un año (citados en Blythe, 1999, p. 
66).Por consiguiente, en el estudio se plantearon metas explícitas en forma de pregunta para que los estudiantes indagaran en sus respuestas a lo largo del trimestre, se hicieran públicas no solo a la clase sino a los padres de familia con la intensión de escuchar opiniones y llamar la atención en el cumplimiento y alcance de la misma, teniendo relación con lo que postula Martha Stone: "las metas de comprensión son especialmente poderosas si se hacen explícitas y públicas" (Stone, 2008).

De igual manera, los desempeños de comprensión fueron establecidos como las actividades o tareas verdaderas que los estudiantes debían realizar para que demostraran sus habilidades; para Tina Blythe y Dorothy Gould, los mejores desempeños de comprensión son aquellos que les exigen a los estudiantes: ir más allá de la información dada con el propósito de crear algo nuevo reconfigurando, expandiendo y aplicando lo que ya saben, así como extrapolando y construyendo a partir de esos conocimientos (citados en Blythe, 2008. p. 88). En esta investigación las actividades planteadas dentro de los desempeños permitieron promover el pensamiento y el desarrollo de la comprensión en los estudiantes usando rutinas de pensamiento, ejercicios de observación y proyectos de aula.

Finalmente, es importante documentar todos los procesos de aprendizaje; es decir, tomar evidencias de todo aquello que realizan los estudiantes para que sea analizado, evaluado y estudiado con más detalle, para así mejorar las prácticas pedagógicas cada día.La visibilidad que ofrece la documentación es la base para la reflexión del propio aprendizaje (Ritcchart et al, 2014 p.52), logrando fortalecer en los docentes la escucha activa, que promueve la buena comunicación y desarrolla habilidades para expresar el pensamiento de una manera más adecuada.

\section{Referencias}

Blythe T. y colaboradores (1999). La Enseñanza para la Comprensión. Guía para el Docente, Buenos Aires, Argentina: Paidós.

Instituto Colombiano para la Evaluación de la Educación (2014). Resultados pruebas saber. Institución Educativa Departamental El Volcán. Ubaté. Cundinamarca.

Ritchhart, R.; Church, M. y Morrison, K. (2014). Hacer visible el pensamiento. Buenos Aires: Paidós. Santelices, L. (1989). Metodología de Ciencias Naturales para la Enseñanza Básica. Santiago de Chile: Andrés Bello.

Stone, M. (. (2008). La enseñanza para la comprensión. Buenos Aires: PAIDÓS. 